\title{
Cable Force-Balancing Distribution of the Cable-Driven Parallel Mechanism for Actuator Saturation Avoidance
}

\author{
Youngsu Cho' ${ }^{1}$, Joono Cheong ${ }^{1}$, Byung-Ju Yi², and Wheekuk Kim ${ }^{1}$ \\ ${ }^{1}$ Korea University at Sejong \\ Sejong-ro 2511, Sejong, Korea \\ Youngsucho@korea.ac.kr, Jncheong@korea.ac.kr, Wheekuk@korea.ac.kr \\ ${ }^{2}$ Hanyang University \\ Ansan, Kyung-gi-do, Korea \\ Bj@hanyang.ac.kr
}

\section{Extended Abstract}

When any actuation cable force of the cable-driven parallel mechanism(CDPM) becomes negative during its operation, the cable goes slack losing its role of force transmission. Thus, every cable tensile force of the CDPM should always be kept positive in order for the CDPM to be properly operational. Indeed, the optimization problem for the cable force distribution of the CDPM should be defined by an appropriate objective function subject to inequality conditions to secure that every cable force is kept within the range, between the minimum and maximum tensile cable forces [1-5]. Recently, the pseudoinverse solution equation for the redundantly actuated PM with one redundant actuation is interpreted as line equations. Then through the search for the intersection points between lines, the computational efficiency in finding the optimal $\infty$-norm solution could be improved significantly [6].

In this work, an optimal cable force distribution algorithm is proposed, which can efficiently search for the minimum $\|f\|_{\infty}$ of the CDPM with two redundant actuations suitable for actuator saturation avoidance. The method is effective to avoid actuator saturation and can be summarized as follows. Force equation of the $n$ DOF CDPM with $n+r$ cables can be expressed as follows:

$$
\boldsymbol{F}_{u}=J_{f} \boldsymbol{f}, \boldsymbol{F}_{u} \in R^{n \times 1}, \boldsymbol{f} \in R^{(n+r) \times 1}, J_{f} \in R^{n \times(n+r)}
$$

where $\boldsymbol{F}_{u}=\left(\begin{array}{llllll}F_{x} & F_{y} & F_{z} & \tau_{x} & \tau_{y} & \tau_{z}\end{array}\right)^{T}$ and $\boldsymbol{f}=\left(\begin{array}{llll}f_{1} & \cdots & f_{n+r}\end{array}\right)^{T}$ denotes the output force vector and the cable force vector, respectively. And $r$ denotes the number of redundant actuations. Assuming that the minimum cable force required to keep the cable taut is $f_{i \min }$, the problem of finding minimal cable force $\left(f_{\text {opt }}\right)$ can be expressed as follows:

$$
\min \|\boldsymbol{f}\|_{\infty} \text { subject to } \boldsymbol{F}_{u}=J_{f} \boldsymbol{f} \text { and } f_{i} \geq f_{i \min }, \text { for } i=1, \cdots, n+r
$$

Note that the general equation for cable force solutions can be obtained by taking the pseudo-inverse of (1) as follows:

$$
\boldsymbol{f}=J_{f}^{+} \boldsymbol{F}_{u}+\left(I-J_{f}^{+} J_{f}\right) \boldsymbol{\varepsilon}
$$

where $J_{f}^{+}=J_{f}^{T}\left(J_{f} J_{f}^{T}\right)^{-1}$ and $\varepsilon$ is an arbitrary vector to be determined. $J_{f}^{+} \boldsymbol{F}_{u}$ is the particular cable force solution and the second term $\left(I-J_{f}^{+} J_{f}\right) \varepsilon$ is the homogeneous cable force solution. By employing the null space vectors the cable force solution could be represented as below: 


$$
\boldsymbol{f}=\boldsymbol{J}_{f}^{+} \boldsymbol{F}_{u}+\sum_{i=1}^{r} \alpha_{i} \boldsymbol{\eta}_{i}=\boldsymbol{f}_{p}+\sum_{i=1}^{r} \alpha_{i} \boldsymbol{\eta}_{i}
$$

where $\boldsymbol{f}_{p}=\left(\begin{array}{lll}f_{p 1} & \cdots & f_{p(n+r)}\end{array}\right)^{T}$ denotes the particular cable force solution, $\boldsymbol{J}_{f}^{+} \boldsymbol{F}_{u} \cdot \alpha_{i}$ and $\boldsymbol{\eta}_{i}($ for $i=1,2, \cdots, r)$ denotes the arbitrary constant to be determined and the null space vector which is defined as $\eta_{i}=\left(\begin{array}{llll}\eta_{i 1} & \eta_{i 2} & \cdots & \eta_{i(n+r)}\end{array}\right)^{T}$, respectively. Note that when $r=1$, (4) can be interpreted as lines, and when $r=2$, (4) can be interpreted as flat planes in the three dimensional space. Thus, the optimal solution, $f_{\text {opt }}=\min \|\boldsymbol{f}\|_{\infty}$, which minimizes the maximal cable force could be analyzed based on the geometric interpretations of those lines or planes.

Indeed, as shown in [6], the optimal solution for the cable actuation force can be found through the search for the intersection points or intersection lines between lines and/or planes. But differently from in [6], additional planes, $f_{p i}+\sum_{j=1}^{r} \alpha_{j} \eta_{j i}=f_{i \min }$, representing the boundary conditions of $f_{i} \geq f_{i \min }, \forall i=, 1,2, \cdots, n+r$ for the CDPM are taken into account in the searching process of the proposed method. Note that (4) can be interpreted as a scalar function of the cable force in the $\alpha_{1}-\alpha_{2}$ plane, $\left\|f\left(\alpha_{1}, \alpha_{2}\right)\right\|_{\infty}$. Thus, the search can be conducted to find the optimal position in the $\alpha_{1}-\alpha_{2}$ plane. The planes corresponding to the conditions $\left(f_{i}=f_{i \text { min }}\right)$ and the intersection lines between two planes corresponding to $f_{i}$ and $f_{j}$ can be mapped into the $\alpha_{1}-\alpha_{2}$ plane as the lines ( $L_{f_{\text {inin }}}$ and $L_{i j}$, respectively).

The searching process can be summarized as follows. In Step 1, as in (4), compute the pseudo-inverse solution (all $f_{p i}$ 's) and two null vectors $\left(\alpha_{1}\right.$ and $\left.\alpha_{2}\right)$. In Step 2, At all the intersection points among the lines $L_{f_{\text {inin }}}$, their infinite norms $\left\|\boldsymbol{f}\left(\alpha_{1}, \alpha_{2}\right)\right\|_{\infty}$ are computed and compared. In this process those intersection points at which any of its cable forces do not satisfy the cable force limit condition of $f_{i} \geq f_{i \min }, \forall i=, 1,2, \cdots, n+r$ are not compared and neglected. The next searching reference point is selected as the one having the minimal value of $\left\|\boldsymbol{f}\left(\alpha_{1}, \alpha_{2}\right)\right\|_{\infty}$ among all the intersection points between the $L_{f_{\text {min }}}$ lines. Note that the next searching reference point always lie on the boundary of the valid cable force solution region after Step 2. In Step 3, compare the values of $\left\|\boldsymbol{f}\left(\alpha_{1}, \alpha_{2}\right)\right\|_{\infty}$ at the intersection points with all the lines on which the current searching reference point lies and select the point at which $\left\|\boldsymbol{f}\left(\alpha_{1}, \alpha_{2}\right)\right\|_{\infty}$ is minimum among them as the next searching reference point. And continue Step 3 until the condition that $\left\|f\left(\alpha_{1}, \alpha_{2}\right)\right\|_{\infty}$ at the current searching reference point is minimal compared with ones at other intersection points is met. Note that if the condition is met, the current searching reference point is the optimal point.

The proposed method is applied to the 3T1R type 4-DOF CDPM having two redundant actuations [7] to compute optimal solutions at the selected configurations with the specified output force at the end-effector of the CDPM within feasible workspace of the CDPM. The optimal solutions are compared with the ones from both LMI and Interior point based optimal methods for verification. It can be confirmed, from these simulations, i) that the same optimal solutions are successfully searched from those three methods, ii) that the computational efficiency of the proposed method is significantly improved compared to the ones of the other two methods, and iii) that the proposed method can be applied to the CDPM in real time applications.

\section{Acknowledgement}

This research was in part supported by Basic Science Research Program through the National Research Foundation of Korea (NRF) funded by the Ministry of Education, Science and Technology (NRF-2015R1D1A1A01061193).

\section{References}

[1] S. Fang, D. Franiza, M. Tordo, F. Bakes, and M. Hiller, "Motion control of a tendon-based parallel manipulator using optimal tension distribution,” IEEE/ASME Trans. Mechatron, vol. 9, no. 3, pp. 561-568, 2004. 
[2] P. H. Borgstrom, B. L. Jordan, G. S. Sukhatme, M. A. Batalin, and W. J. Daiser, "Rapid computation of optimally safe tension distributions for parallel cable-driven robots," IEEE Trans. On Robotics, vol. 25, no. 6, pp. 1271-1281, 2009.

[3] A. Pott, T. Bruckmann, and L. Mikelsons, "Closed-form force distribution for parallel wire robots," in Computational kinematics, Springer-Verlag Duisburg, Germany, 2009, pp. 25-34.

[4] L. Mikelsons, T. Bruckmann, M. Hiller, and D. Schramm, "A real-time capable force calculation algorithm for redundant tendon-based parallel manipulators," in Proceedings of International conference on robotics and automation, 2008, pp.3869-3874.

[5] J. Lamaury and M. A. Gouttefarde, "A Tension Distribution Method with Improved Computational Efficiency," In Cable-Driven Parallel Robots, vol. 12, pp. 71-85, 2013.

[6] S. H. Woo, S. M. Kim, M. G. Kim, B.-J. Yi, and W. K. Kim, "Torque-balancing Algorithm for the Redundantly Actuated Parallel Mechanism," Mechatronics, vol. 42, pp. 41-51, 2017.

[7] H. B. Cho, S. M. Kim, B. J. Yi, W. K. Kim, "Workspace Analysis of a Wire-driven 3T1R Type Haptic Mechanism," in Proceedings of 2016 KSPE spring conference, Korea, 2016, pp. 279-280. 